\title{
Electron Tunneling and Electrochemical Currents through Interfacial Water Inside an STM Junction
}

\author{
Moon-Bong Song, Jai-Man Jang, and Chi-Woo Lee* \\ Department of A hanced Haterials Chemistry, Korea Cniversitw, Jochin on, Choongnam 339-700, Korea \\ Received Julv 16,2001
}

\begin{abstract}
The apparent barrier height for charge transfer through an interfacial water layer between a $\mathrm{Pt} / \mathrm{Tr}$ tip and a gold surface has been measured using STM technique. The average thickness of the interfacial water layer inside an STM junction was controlled by the amount of moisture. A thin water layer on the surface was formed when relative humidity was in the range of 10 to $80 \%$. In such a case, electron tumneling through the thin water layer became the majority of charge transfers. The value of the barrier height for the electron tumneling was determined to be $0.95 \mathrm{eV}$ from the current vs. distance curve. which was independent of the tip-sample distance. On the other hand the apparent barrier height for charge transfer showed a dependence on tip-sample distance in the bias range of $0.1-0.5 \mathrm{~V}$ at a relative humidity of approximately $96 \%$. The non-exponentiality for current decay under these conditions has been explained in terms of electron tunneling and electrochemical processes. In addition. the plateau current was observed at a large tip-sample distance, which was caused by electrochemical processes and was dependent on the applied voltage.
\end{abstract}

Keywords : STM, Electron tunneling. Electrochemical current. Barrier height.

\section{Introduction}

Charge transfer through a condensed phase is of fundamental inportance to many processes in the surface science and electrochemistry. ${ }^{1-15}$ A characteristic of direct electron tumneling has been extensively studied among charge transfers using scanning tunneling nicroscopy (STM) under ultrahigh vacuun (UHV) circuntstances ${ }^{2-5}$ A value of an apparent barrier height for tumneling related to the local wave function is easily deternined from conductance variation with tip-surface distance. From a simple one dimensional turneling model as well as experimental studies. the barrier height was found to be independent of the bias voltage applied between an STM tip and a surface, and of the surface-tip spacing at longer distances than that coning into a quantum-point contact. ${ }^{-5}$

In a similar way, attempts to measure STM barriers have been made in humid atmosphere and electrochenical environment. ${ }^{5-15}$ Such enviromments give rise to thin and thick water layers, respectively, inside the region between an STM tip and a surface. Therefore. mechanism of charge transfers across interfacial water layer in ambient conditions is not so simple as compared with that observed in UHV and is still not well understood. Furthermore. the electrochemical effects have discouraged us from acquiring detailed information about electron tumeling in an aqueous phase. It was reported that the turneling distance increases because of the presence of water layers inside an STM junction in both humid atmosphere and electrochemical environment. ${ }^{6.79 .14 .16}$ The value of the local barrier height for electron tumeling was low compared with the UHV case and shows bias dependence as well as tip-surface distance dependence. ${ }^{6.79 .14 .16}$ In this work, we examined charge transfer through the interfacial water layer inside an STM junction, of which the average thickness was controlled by the amount of moisture The gold/water interface was chosen as a model system to investigate the single crystalline silicon/water interface. We also investigated current behaviors as a function of the applied bias voltage.

\section{Experimental Section}

The experiment was carried out using a PicoSPM (Molecular Inaging Corp.) at a temperature of $20( \pm 1)^{\circ} \mathrm{C}$. We used Au substrates deposited onto mica and annealed them in a hydrogen flame for $1 \mathrm{~min}$ prior to use. Platinum/ridium tips were prepared mechanically from $\mathrm{Pt} / \mathrm{Ir}$ wire. A sample stage was contained in an environmental chamber, where a hygrometer and a thermometer were placed to check the equilibrium humidity and temperature, respectively. The relative humidity below $10 \%$ inside the chamber was kept by silica desiccant. Higher relative humidity was obtained by means of the careful exposure of wet paper for a few seconds, while wet paper was introduced into the chamber to reach near saturated condition. The chamber was initially flushed with dry nitrogen gas. The system was left for $1-10$ hours to stabilize relative humidity and avoid themmal drift before the experiment.

Current $1 s$. distance characteristics were measured in the following way: an initial current was held at $5 \mathrm{nA}$ by using a constant current feedback. The bias voltage was applied to the tip and was adjusted to the fixed value that was selected in the range between -0.5 and $0.5 \mathrm{~V}$. To make a measurement. the tip was pushed by $0.3 \mathrm{~nm}$ towards the sample after the feedback was switched off. Then, the tip was rapidly withdrawn from the sample and the resulting current in the range between 10 and $0.001 \mathrm{nA}$ was recorded as a function of the distance perpendicular to the surface. Afterward, the 
tip was moved back to its original position, and the feedback loop was enabled again. For each bias voltage value, this process was repeated 20-60 times. and then the data were averaged. The data were found to give sufficiently reproducible results.

\section{Results and Discussion}

Figure 1 shows the typical current $w$. tip distance data measured on a gold surface evaporated onto mica with different relative humidities and tip bias voltages. Solid circles were obtained at a low relative humidity of $10 \%$ and at a bias voltage of $0.1 \mathrm{~V}$. The tunneling current data are well fitted by a single exponential decay curve (indicated by the solid line) with the apparent barrier height value of $0.95 \mathrm{eV}$ as a function of the tip-surface distance. Note that the tip displacement represents the displacement from the equilibrium tunneling distance at $I_{t}=5 \mathrm{nA}$. In a simple one dimensional tumneling model, ${ }^{1}$ the tunneling current (I) inside an STM junction can be written as $I=I_{0} \exp (-1.025 \sqrt{\phi \cdot s})$. where $\phi$ is the apparent barrier height in electron-rolts and $s$ the distance in angstroms. The value of $0.95 \mathrm{eV}$ was found to be almost constant. irrespective of the applied bias voltage over the range between -0.5 and $0.5 \mathrm{~V}$ at the relative humidity extending from 10 to $80 \%$. Our measured value is low compared with that in ultrahigh vacuum. The difference

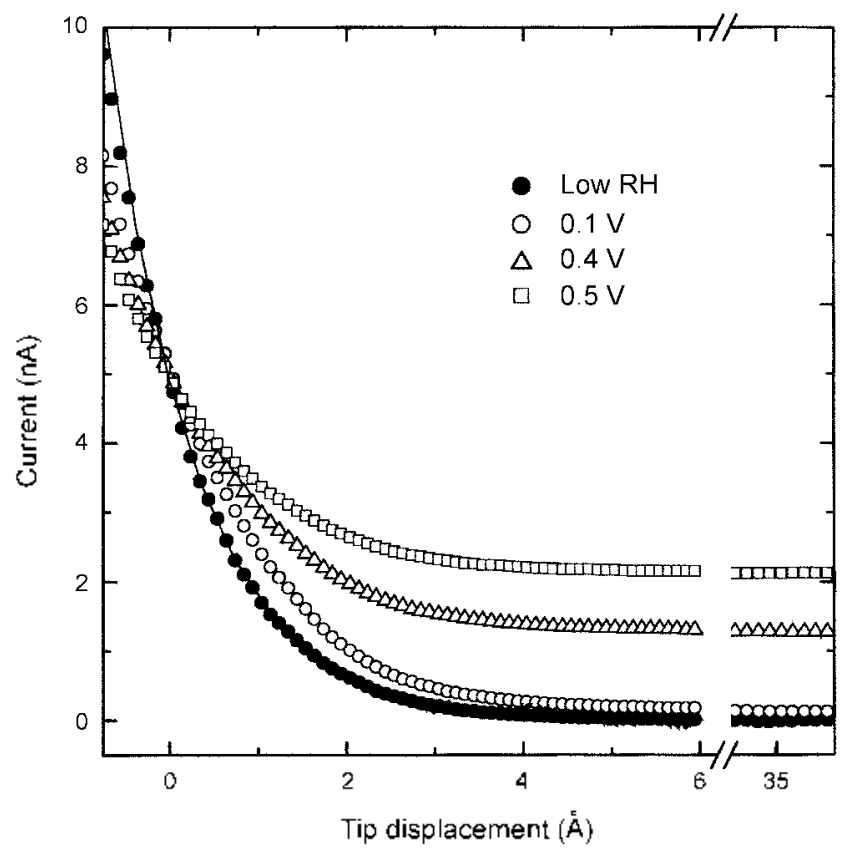

Figure 1. Cument versus distance data measured on an Au surface with a Pt/It tip in air with different relative humidities and tip bias voltages. The tunneling current data, which were recorded at a relative humidity below $10 \%$ at a bias of $0.1 \mathrm{~V}$, were indicated as solid circles. Open circles, triangles and squares were obtained at bias voltages of $0.1,0.4$ and $0.5 \mathrm{~V}$, respectively, at a relative humidity as high as about $95 \%$. Note that the tip displacement represents the distance from the equilibrium tunneling distance at $I_{t}$ $=5 \mathrm{nA}$. The solid curve is exponential fitted with the apparent barrier height of $0.95 \mathrm{eV}$. in the tunneling barrier height can be explained in terms of the difference of tumneling environment ${ }^{6.7 .914 .16}$ That is. the electron tumneling under ultrahigh vacuum and humid atmosphere takes place directly and across thin layer of water on the surface. respectively.

It is well known that the apparent barrier height is independent of the tip-surface separation. ${ }^{1}$ Therefore. the width of a tunnel gap can be estimated by extrapolating the conductance to the expected value at quantum-point contact $(77.52 \mu \mathrm{mho}){ }^{11,14}$ In the present work, the tumnel distance on the gold surface is calculated to be $12 \pm 1 \AA$ from the tumneling current of the solid circles in Figure 1 . The value for the apparent barrier height and the tumnel distance are consistent with the previous STM results ${ }^{6,7}$ studied under ambient atmosphere with $50-80 \%$ relative humidity. Also, independence of the apparent barrier height from the applied bias voltage and polarity in the tunnel current range less than $10 \mathrm{nA}$ is in good agreement with the STM result. ${ }^{6}$.

Current decay data recorded for a relative humidity as high as about $96 \%$ (using an uncoated tip) are also shown as open circles. triangles and squares at the bias voltages of 0.1 , 0.4 and 0.5 V. respectively. in Figure 1. Humidity increases the thickness of the water pillar formed inside an STM junction. An important feature that is different for two humidities is the presence of the plateau current at larger tipsurface separation. At a distance range longer than $5 \AA$ where the current decays are approximately completed. the current has a certain constant value depending on the applied bias voltage. The plateau currents were observed to present hysteresis in the direction of the tip movement. The plateau at $0.5 \mathrm{~V}$ bias was extended to a length of one hundred of nanometers during the tip retraction. However, the width of the plateau on the approaching movement was substantially shorter. The plateau currents started to appear at a relative humidity above $92 \%$, and its magnitude was found to strongly depend on the relative humidity. The values of the plateau currents measured as a function of the bias voltage are plotted in Figure 2. Data were obtained at the relative humidity of $96 \%$. Each point is an average of many experiments that are indicated by the standard deviations. The plateau current increased as the difference in bias voltage between the tip and the sample increased.

The present result is compatible with the previous work. ${ }^{\text {? }}$ The plateau current was reported to be found with the hydrophilic surfaces such as $\mathrm{Pt} / \mathrm{C}$ and mica at the relative humidities of $50-80 \%$ and above $65 \%$. respectively at large bias voltage. ${ }^{9}$ However. it was not detected on a gold surface up to a relative humidity of $75 \%$ because of its hydrophobicity. ${ }^{9}$ The presence of the plateau was generally interpreted by the formation of the thick layer of water with conductivity. We found from our quartz crystal microbalance experiments that the average thickness of water layer on the gold surface is of the order of nanometer near saturated relative humidity. ${ }^{17}$ When the tip is approached toward the sample surface. it comes into contact with the conductive water layer. This results in an electrochemical current being dependent on the applied voltage. On the contrary. the water 


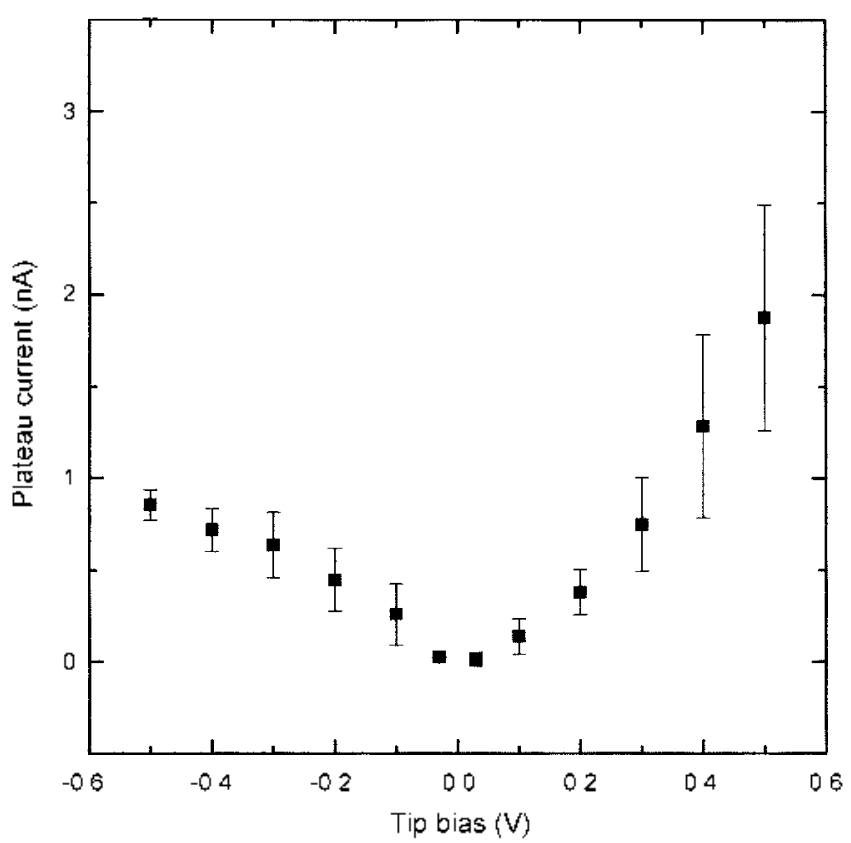

Figure 2. Electrochemical plateau curent as a function of bias voltage. Data were obtained at relative humidity of $96 \%$. Each point is the average of more than 20 experiments with the standard deviations.

bridge formed between the tip and the sample is elongated due to water meniscus adhered to the tip during the tip retracting. Consequently. such a phenomenon leads to the different widths of the plateau depending on the direction of the tip movement.

On the other hand, an asymmetry with respect to the applied bias polarity is clearly observed in Figure 2 . That is, the height of the plateau current on a negatively charged surface is higher than that on a positively charged surface. The plateau currents showed a strong tendency to asymmetry at high bias voltages $(>0.3 \mathrm{~V})$. whereas they were almost independent of the polarity at lower biases $(<0.3 \mathrm{~V})$. The reason for this may be inferred from the fact that the electrochemical reactivity depends on the surface property of electrode material. Two electrode materials used to form an STM junction in the present study are Au and Pt/Ir. Such a difference in these two electrode materials gives rise to different electrochenical reactivities leading to the asymmetry of plateau current at large bias potentials. The asymmetry of these plateau currents may not be differentiated under a low extennal electric field because the difference of the materials will not be sufficiently effective at the low bias.

Now. we discuss current decay for charge transfer at a high relative humidity. Figure 3 shows the values of the barrier height as a function of tip distance. which was obtained by subtracting the constant plateau current caused by electrochemical processes at a given bias voltage from its original current decay. Solid circles were obtained at the relative humidity of $10 \%$ with the bias voltage of $0.1 \mathrm{~V}$, and open circles, triangles and squares at the relative humidity of $96 \%$ with the bias voltages of 0.03 .0 .1 and $0.4 \mathrm{~V}$, respectively. The apparent barrier height of $0.95 \mathrm{eV}$ for higher

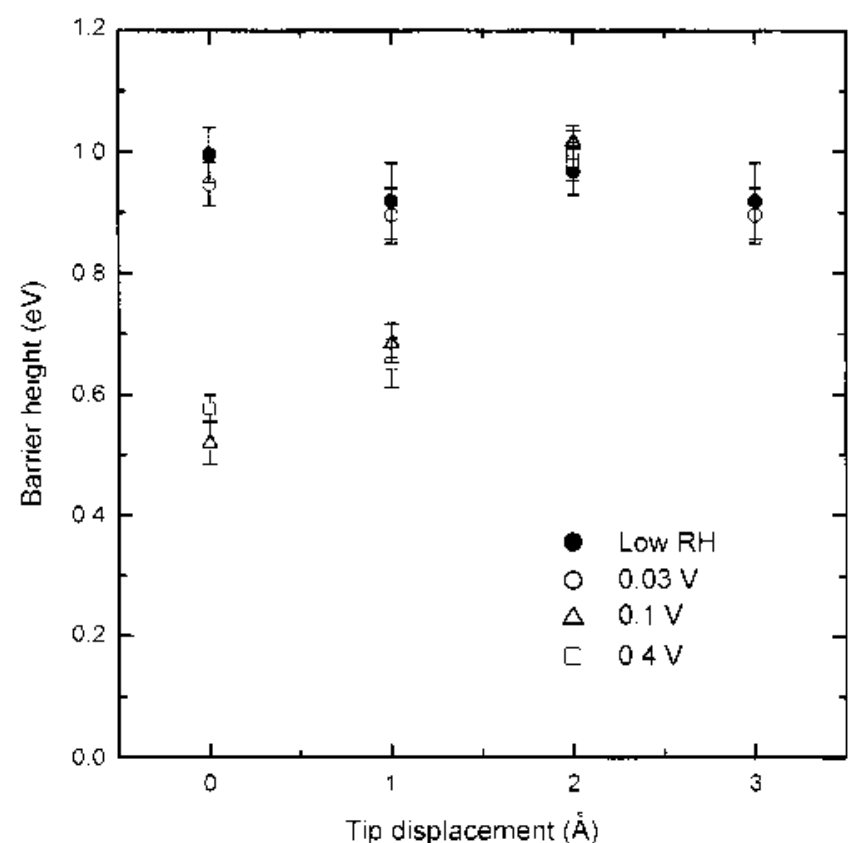

Figure 3. Apparent barrier heights for charge transfer as a function of the tip distance after each constant platean cument caused by electrochemical processes at a given bias voltage was subtracted from its original current decav. Solid circles were obtained at a relative humidity of $10 \%$ at a bias voltage of $0.1 \mathrm{~V}$, and open circles, triangles and squares at a relative humidity of $96 \%$ with bias voltages of $0.03,0.1$ and $0.4 \mathrm{~V}$, respectively. The "zero" tip displacement corresponds to the equilibrium tunneling condition at $I_{t}=5 \mathrm{nA}$.

humidity with $0.03 \mathrm{~V}$ (open circles) is equivalent to that measured for the low humidity (solid circles) within the experimental uncertainty. Also. the tip-surface spacing dependence of the barrier height was not observed. Since electrochemical processes are not taking place rapidly under such a low electric field and the tip distance becomes shorter. a plateau current that results from the electrochemical processes could not be detected over the long distance. From these results, it can be concluded that the current at the low bias is attributed to electron tunneling through the water layer inside the STM junction in spite of a high relative humidity

One of the most important features on current-yoltage characteristics concems the current decay on the surface with thick water layer at ligh voltages. The apparent barrier heights for $0.1-0.5 \mathrm{~V}$ show the tip-distance dependence as indicated in Figure 3. At a tip distance of $2 \AA$ the value is about $1.0 \mathrm{eV}$. decreasing to about $0.55 \mathrm{eV}$ when the tip distance was reduced to $0 \AA$. The result implies a nonexponential decay of current, contrasted with the exponentiality observed for (the low and higher humidities with) a low bias voltage. Such a phenomenon was previously reported to be observed in water ${ }^{14}$ as well as in UHV. ${ }^{4.18}$ A non-exponentiality in vacuum tumneling is a consequence of quantum point contact within the distance of a few angstroms from the surface. In a $\mathrm{HClO}_{4}$ solution, the tunnel distance between a $\mathrm{Pt} / \mathrm{Ir}$ tip and a $\mathrm{Au}(\mathrm{lll})$ surface was calculated to be 
approximately $20{ }^{14}$ Also, the tumeling barriers were found to be 1 and $0.25 \mathrm{eV}$ at tunneling resistances of $10^{19}$ and $10^{8} \Omega$ respectively. ${ }^{1+}$ The trend that the value decreases with a decrease of the tip distance is compatible with our result. even though the absolute tumel distances can not be compared for the two experiments.

It has been suggested in previous reports that the change in barrier height could be caused by electron tunneling through thick water vic some intermediate states such as resonant states. changes of water structures near a electrode. dry or wet state with hydrated electron. localized electronic state on the water molecules and quantum mechanics of water clusters. ${ }^{9,10,14}$ The effect of electrochenical currents on charge transfer has been ignored in previous studies. We believe that the effect of electrochenical currents on charge transfers has not been noticed because nearly completely coated tips except the apex atom have been employed in previous studies. However, some contributions of electrochemical effects to current decay were experimentally hinted at extreme potentials in previous studies. ${ }^{8,9} \mathrm{~A}$ ligh resolution image could be obtained using electrochenical reactions. ${ }^{8}$ That is. the electrochemical current varies depending on the gap distance. An observation of atomic resolution in electrochemical STM operated at relatively large gap distance (20) could not be explained by electron tunneling only. ${ }^{1+}$ The apex atom on a tip has a large capacity to pass current of the order of several hundreds of microamperes in the bias range of $0.1-0.5 \mathrm{~V}$, as calculated from quantum-point contact. ${ }^{11.14}$ In the present investigation, we used an uncoated tip and was able to observe electrochemical effects clearly. At high bias voltages electrochemical currents are observed to make a large contribution toward charge transfer as mentioned above. Additionally, we hired a coated tip having an extremely small electrode area to check the dependence of the plateau current on the area of the electrode surface as expected for the electrochemical processes. The magnitude of the plateau current at a tip bias of $0.5 \mathrm{~V}$ was reduced by about a fifth as compared to that for an uncoated tip in the identical conditions. Thus the present results give a strong evidence for the electrochenical current flowing through the interfacial water inside the STM junction. Hence we suggest that the electrochemical current may altematively contribute to current decay. Certainly to elucidate the mechanism of charge transfer through water layer inside the STM junction. it is required to obtain more detailed information about the structure of electric double layer.

\section{Conclusions}

STM technique was used to measure the apparent barrier height through interfacial water between a Pt/Ir tip and a gold surface. The average thichness of the interfacial water layer inside an STM junction was controlled by the anount of moisture. When the water density on the surface was low, i.e., the water layer had a low average thickness. the current was almost exclusively transferred by the electron tunneling. The value of the apparent barrier height of the electron tunneling was determined to be $0.95 \mathrm{eV}$ from the current $\%$. distance curve, which was independent of the tip-sample distance. On the other hand, the distance dependence of the apparent barrier height appeared under the thick interfacial water layer. At a tip distance of $2 \AA$ away from the equilibrium tumeling distance at $5 \mathrm{nA}$. the value was about 1.0 $\mathrm{eV}$. decreasing to about $0.55 \mathrm{eV}$ when the tip distance was reduced to $0 \AA$. The non-exponentiality for current decay has been explained in previous studies in terms of electron tumneling via some intermediate states over the wider gap distance. However, we alternatively suggest the current decay to be caused by electrochemical processes. In addition. the plateau current was observed, which was caused by electrochemical processes and was dependent on the applied voltage.

Acknowledgment. This work was generously supported by Korean Research Foundation Grant through Basic Science Research Institute Program (KRF-2001-015DP0372).

\section{References}

1. Wiesendanger. R. Scaming Probe Mficroscopy and Spectroscopy Methods and Applications: Cambridge: New York. 1994.

2. Schuster. R.: Barth. T. V.: Wintterlin. J: Behun. R. J.: Ertl. G. Utranticroscopy 1992. +2-44. 533.

3. Biscarini. F; Kenkre. V. M. Stoff. Sci. 1999, +26, 336.

4. Olesen. L: Lagggaard, E.: Stensgaard, I.: Besenbacher, F.; Schiotz. J; Stoltze, P.: Jacobsen, K. W; Norskov. J. K. Plws. Rev Letts 1994. 72. 2251

5. Mamin. H. J.: Ganz. E.: Abraham. D. W.: Thomson. R. E.: Clarke. J. Phis Ren: 1986. B34.9015.

6. Hong, Y. A; Hahn. J. R; Kang. H. J. Chem. Plys. 1998. 108. 4367.

7. Hahn, J. R.: Hong, Y. A.: Kang, H. Appl Phys. 1998. 166, S 467.

8. Fan1. F.-R. F.: Bard. A. J. Science 1995. 270.1849.

9. Heimn. M: Eschrich. R: Hillebrand. A: Knapp. H. F. Guckenberger. R.: Cevc. G. J. Tac. Sci. Techmol. 1996. Bt2. 1498.

10. Schmickler. W. Surf. Sci. $1995.335,416$.

11. Pan. J.: Jing. T. W.: Lindsay, S. M. J. Phus. Chem. 1994. 98.4205.

12. Meepagala. S. C.: Real. F. Phus. Rev 1994. B49, 10761

13. Guckenberger. R.: Heim. M.: Cewc. G.: Knapp. H. F.: Weigrabe. W: Hillebrand. A. Science 1994. 266. 1538.

14. Vaught. A.: Ting. T. W.: Lindsay. S. M. Chent. Phis. Letts 1995. 236. 306 .

15. McCarley. R. L.; Hendricks, S. A; Bard, A. J. J. Phys. Chem. 1992. 96,10089

16. Sass. I. K.: Gimzewski. J. K. J. Electromal. Chem. 1991. 308. 333.

17. Song. M. B.: Jang. T. M.: Bae. S. E.: Lee. C. W. accepted for publication in Langmair.

18. Lang. N. D. Phys. Rev 1987, B36.8173.

19. Ahn, J. H: Pyo, M. H. Bull. Kowan Chem Soc. 2000. 21. 64t. 